\title{
Rho and Ras GTPases in semaphorin-mediated neuronal development
}

\author{
Lifei Fan, Morigen \\ College of Life Sciences, Inner Mongolia University, Hohhot, China \\ Email: fanlifei95@gmail.com, morigenm@hotmail.com
}

Received 1 November 2012; revised 4 December 2012; accepted 4 January 2013

\begin{abstract}
Neurons are highly polarized cells with a single long axon and multiple dendrites, all of which are actinrich structures. The precise regulation of neuronal cell morphology is a fundamental aspect of neurobiology. The major role of Rho GTPases, which is conserved in all eukaryotes, is to regulate the actin and microtubule cytoskeleton. Therefore the Rho GTPases are key regulators of neuronal morphology during development besides their canonical functions in actin cytoskeletal regulation, cell migration and cell cycle progression. Semaphorins are a family of secreted or transmembrane proteins, which function through their receptor plexins and/or neuropilins to act as the repulsive or attractive guidance cues for axons and dendrites. It has been demonstrated that the fully activation of plexins appears to be dependent on the binding of Rho GTPases to the Rho binding domain (RBD) and Semaphorin to the extracellular region. Here, we summarize the functions of the small Rho GTPases in two well-studied vertebrate Semaphorins, Sema3A and Sema4D; and the potential roles of the small Rho GTPases in some poorly-studied vertebrate Semaphorins Sema5A, Sema6A and Sema7A. We also summarize the functions of different members of Ras family, R-Ras, M-Ras and Rap, in Semaphorin signalling pathways as well.
\end{abstract}

Keywords: Small Rho GTPase; Semaphorin Signalling; Neurite Development

\section{INTRODUCTION}

The development of a neuron requires a series of steps that begins with the migration from its birth place, the initiation and outgrowth of neurite that serve as precursors to axons and dendrites, the polarization, growth, guidance and branching of axons, the formation of dendrites and spines, and finally the formation of connections that allow a neuron to make communication with appropriate targets [1]. The Rho GTPases have been found to be involved in several neuronal development processes, such as neurite outgrowth and differentiation, axonal initiation, outgrowth, guidance and branching, and dendritic spine formation and maintenance [2,3]. This part will focus on the role of Rho GTPases, Ras GTPases, semaphorins, plexins, and their associated effectors in the regulation of axonal guidance and dendrite growth.

\section{SEMAPHORINS AND RECEPTORS}

\subsection{Semaphorins}

Semaphorins were initially identified as an axonal repellent in the central nervous system development [4]. Over the last few years, it has been shown that, like most guidance cue families, semaphorins have both repulsive and attractive functions; moreover, many semaphorins are bifunctional [5]. Semaphorins can be divided into 8 classes based on their sequence similarity and structural domains contained. The class 1 and class 2 semaphorins are found in invertebrates; classes 3 - 7 are vertebrate semaphorins; and the last class is found in virus. Classes 2 and 3 and the viral semaphorins are secreted; whereas semaphorins belonged to classes 1, 4, 5, 6 and 7 are all cell membrane-anchored proteins. All semaphorins are characterized by the conserved N-terminal sema domain that is essential for the recognition with another sema domain on the semaphorin receptor. The semaphorin domain contains a region that specifies its biological activity, which consists of a highly conserved 70 amino acid variant form of the seven-blade beta-propeller fold $[6,7]$. At the $C$ terminus of sema domain there is another conserved domain named as PSI (plexins, semaphorins and integrins) domain. Different members of semaphorin family are distinguished by unique additional domains besides sema domain and PSI domain, such as a signal immunoglobulin (Ig)-like domain is found in classes 2, 3, 4 and 7 semaphorins, thrombospondin repeats exhibited in class 5 semaphorins, glycosylphosphatidylinositol (GPI) anchor in class 7 semaphorins and PSD-95/Dlg/ZO-1 (PDZ) binding motif at the $\mathrm{C}$ terminus of class 4 sema- 
phorins [8].

\subsection{Semaphorin Receptors}

The predominant receptors for semaphorins are plexins, there are nine members in vertebrates and two in invertebrates. The vertebrate plexins can be subgrouped into four; four type A plexins, three type B plexins, one $\mathrm{C}$ type and one D type. Plexin was first identified as a neuronal cell surface molecule that mediates cell adhesion [9]. Plexins contain a divergent sema domain and followed by three PSI domains and three IPT domains (Ig-like, plexin and transcription factors) in their extracellular region. The plexins are distinguished by the presence of a highly conserved intracellular split GTPase-activating (GAP) cytoplasmic domain [10]. The unusual thing is the GAP-homology domain is divided into two separated parts by an unrelated linker region, which was shown to be able to directly interact with several small Rho GTPases, such as Rac, Rnd1 and RhoD [11-13]. The type B plexins contain an additional C-terminal PDZ domain binding site for the interaction with the PDZ domain containing RhoGEFs [14]; and they also contain a conserved cleavage site in their extracellular domain, which can be cleaved by furin-like proprotein convertases. This post-translational processing greatly increases the binding and the functional response to their specific ligand semaphorin4D [15].

Plexins are autoinhibited in the absence of ligand, binding of semaphorin to the sema domain of plexin releases the autoinhibition between the sema domain and the ectodomain and leads to plexin activation [16]. Classes 4 - 7 semaphorins together with Sema3E from class 3 bind specific plexins directly through sema-sema domain interaction and activate following signalling pathways, whereas the other members of class 3 semaphorins require neuropilins to serve as binding co-receptors and the plexins as the signalling-transduction elements [17].

Neuropilins are transmembrane proteins with short intracellular domains that lack intrinsic enzymatic activity, and the ectodomain is too short to mediate independent signal transduction. Thus neuropilins function as coreceptors to form a transduction complex with other transmembrane receptors. The neuropilin family contains two members; neuropilin 1 (NP1) and neuropilin 2 (NP2). The two kinds of neuropilins share a similar domain structure, although the overall homology at the aminoacid level is only $44 \%$ [18]. Neuropilins are found to act as co-receptors for Semaphorin 3 subfamily, and the binding ability to different members of Semaphorin 3 is varied among NP1 and NP2. For example, Sema3A binds specifically to NP1, whereas Sema3F has high affinity with NP2 compared with NP1 [18,19].

\section{FUNCTIONS OF THE SMALL RHO GTPASES IN SEMA3A-PLEXINA1 AND SEMA4D-PLEXINB1 SIGNALLING PATHWAYS}

Most of our understanding concerning semaphorin signal transduction in corporation with plexin receptors originates from studies of Sema3A and Sema4D in neuronal cells. The growth cone is a sensitive region located at the tip of a developing axon, which can sense the extracellular cues. In response to attractive and repulsive signals, there is continuous reorganization of actin cytoskeleton within lamellipodia and filopodia that accounts for growth cone guidance. Dendrites extend from the cell body to form a multi-branched tree-like structure, which receive, process, integrate synaptic inputs from other neurons [20].

Sema3A, a secreted semaphorin, acts as an attractive or a repulsive guidance cue for axons by activating a receptor complex containing NP1 and plexinA1 to work as the ligand-binding subunit and the signal-transducing subunit, respectively. In the steady state, plexinA1 is antoinhibited and is associated with NP1 [16]. FARP2, a GEF for Rac, is associated with the plexinA1/NP1 complex [21,22]. The binding of Sema3A to plexinA1/NP1 complex not only causes the release of the plexinA1 auto-inhibition but also leads the dissociation of FARP2 from the complex and activation of its Rac-GEF activity. GTP-bound form of Rac is sequestered by plexinA1 and in turn causes the recruitment of another Rho GTPase Rnd1 to the linker region of intracellular domain of plexinA1. Binding of Rnd1 to the linker region disrupts an inhibitory interaction between the N-terminal and C-terminal parts of the split GAP domain [23]; then the intrinsic R-Ras GAP activity of the split GAP domain of plexinA1 is activated, followed by the downregulation of R-Ras activity, the inactivation of integrin, the turnover of focal adhesion and the detachment of cells from the ECM [22]. GTP-bound Rac can activate PAK to initiate actin polymerization, so the sequestration of RacGTP inhibits PAK activation, which therefore inhibits actin polymerization. In another aspect, the FERM domain of FARP2 sequesters an isoform of type-I phosphatidylinositol phosphate kinase, PIPKI $\gamma 661$, inhibiting its interaction with focal adhesion protein talin and suppressing focal adhesion. The Sema3A/plexinA1 signalling pathway influences focal adhesion property in three ways; sequestration of Rac, downregulation of R-Ras and sequestration of PIPKI $\gamma 66$, all of which lead to suppression of integrin functions and inhibit cell adhesion to the extracellular matrix, and ultimately cause the axonal repulsion [22]. Interestingly, the interaction between Rnd1 and plexinA1 can be inhibited by RhoD, which binds to the same linker region as Rnd1 does on plexinA1 [11,24] (Figure 1). 


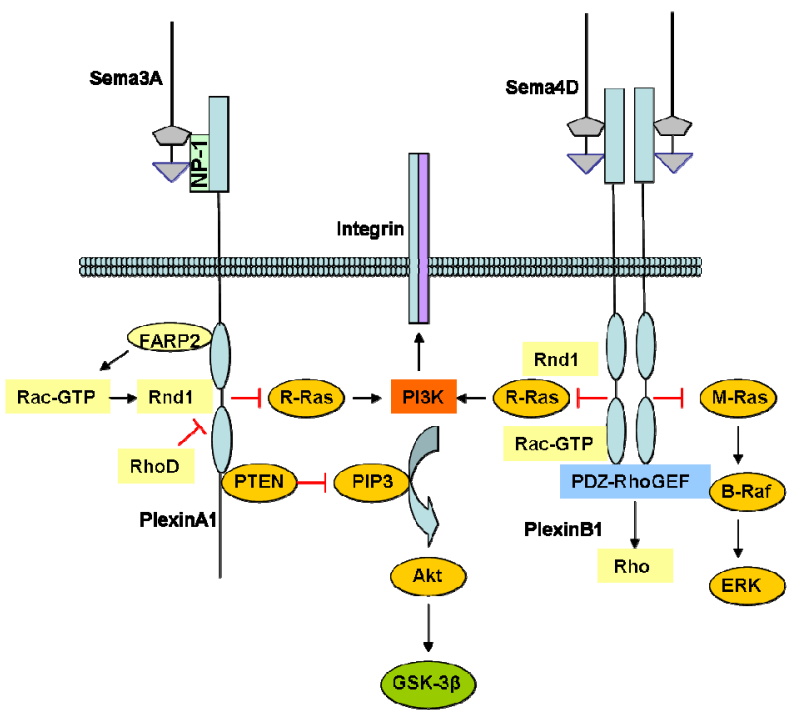

Figure 1. Semaphorin intracellular signalling events. The main signal transduction pathways by which Sema3A and Sema4D active plexinA1 or plexinB1. Plexin-mediated effects on actin cytoskeletal remodelling are regulated by Rho GTPases; Rac1, Rnd1 and RhoD. Semaphorin signalling results in growth cone repulsion in the nervous system development due to the activation of the R-Ras GAP activity of the intracellular domain of plexinA1 and plexinB1, and in turn inhibits the integrin function through suppressing PI3K signalling pathway. Semaphorin signalling results in dendrite repellence in the nervous system development due to the activation of the M-Ras GAP activity of the intracellular domain of plexinB1, and in turn suppresses ERK activation. PleixnB1 induces axonal growth cone collapse and dendrite repellence through its Ras-GAP activity for R-Ras and M-Ras, respectively (Figure adapted from [5]).

Sema4D, also known as CD100, is a transmembrane semaphorin that works through plexinB1 as its receptor. It has been shown that a 120-residue cytoplasmic independent folding domain of plexin-B1 that directly binds three Rho family GTPases, Rac1, Rnd1, and RhoD; and the Cdc42/Rac interactive binding-like motif of plexinB1 is not involved in this interaction [11]. In the resting state, plexinB1 are dimerized and inactive, the binding of Sema4 4 to the receptor causes the conformational change and leads to the recruitment of Rac1 and Rnd1 to the plexinB1. The dimeric structures are altered and the plexinB1 is activated. The intrinsic R-Ras GAP activity of the split GAP domain of plexinB1 is also activated by the binding of Rac1 and Rnd1 to the liker region, which leads to the inhibition of R-Ras activity and focal adhesion turnover [10]. The binding of RhoD to the Rho GTPase interacting region inhibits Rnd1 activity. Compared to other semaphorins, type B semaphorins have a C-terminal PDZ binding domain that can directly interact with PDZ-RhoGEF/LARG, a Rho GEF, to induce RhoA and ROCK activation [14]. Surprisingly, the cell contraction triggered by Sema4D and plexinB1 signalling pathway is both Rac1 and RhoA dependent, but how
Sema4D induces Rac1 activation is still unclear [12] (Figure 1).

\section{POTENTIAL FUNCTIONS OF THE SMALL RHO GTPASES IN OTHER VERTEBRATE SEMAPHORIN SIGNALLING PATHWAYS}

Sema5A is a transmembrane semaphorin, and the unique feature of class 5 semaphorins is that they contain seven thrombospondin repeats in their extracellular region. It has been shown that Sema5A interacts specifically with plexinB3, and the thrombospondin repeats may play indispensable roles in this interaction, as truncated form of Sema5A comprising the sema domain but lacking thrombospondin repeats interacts weakly with plexinB3 [25]. Sema5B is the highly homologous Semaphorin with Sema5A; however, it does not interact with plexinB3. On the other hand, Sema5A does not interact with either plexinA1, plexinB1 or plexinB2 [25]. Recently, It has been shown that Sema5A inhibits human glioma cell motility through the complex formed by its receptor plexinB3, RhoGDI $\alpha$ and Rac1 [26]. The cytoplasmic domain of plexinB3 interacts with RhoGDI $\alpha$ directly; after Sema5A binding to plexinB3, both RhoGDI $\alpha$ and active Rac1 are recruited to plexinB3 in a noncompetitive manner, providing a docking site for their interaction. The interaction between RhoGDI $\alpha$ and plexinB3 is transient, after a short time, RhoGDI $\alpha$ dissociates from plexinB3 together with active Rac1; therefore Rac1 is extracted from cell membrane by RhoGDI $\alpha$ and the Rac1 signal is silenced. It was also demonstrated that the enhanced interaction between RhoGDI $\alpha$ and Rac1 under Sema5A stimulation is dependent on plexinB3 [26]. This is another example of how Semaphorin and their plexin receptors regulate actin cytoskeleton through Rho GTPases and their regulators. Intriguingly, small Rho GTPases such as RhoA and Cdc42 can not bind to plexinB3; it will be of great interest to investigate whether Rnd1, RhoD or other small Rho GTPases could be able to interact with plexinB3, and the contribution of the splited Ras GAP domain of plexinB3 on the downstream signalling.

Sema6A, a transmembrane semaphorin, was first identified as a repulsive axon guidance cue [27]. It has been shown recently to promote the dendritic growth of lateral motor column (LMC) neurons through Sema6A-plexinA4-FARP1 signalling pathway [28]. In fact, Sema3A can also repel growth cone while under other conditions stimulates the dendrite outgrowth [29]. FARP1, also named as CDEP, is comprised of an N-terminal FERM domain, a central DH domain, and the C-terminal PH1, PH2 domains. The DH-PH1 domain is conserved in all eukaryotic GEFs, and the PH domain is responsible for binding to the membrane or cytoskeleton [30,31]. Al- 
though FARP1 shares similar domain structures with FARP2, they two have distinct functions in triggering the exchange of GDP to GTP; FARP2 is a specific Rac1 GEF [21], while FARP1 is a GEF for Rho [31]. FARP2 has been shown to be involved in cell-cell junction formation downstream of nectins and in axonal repulsion downstream of semaphorin 3A [22]. To date very little is known about FARP1; it is upregulated in differentiating chondrocytes, and is expressed in liver, spleen, brain, heart and intestine [31]. It is found that FARP1, a specific effector for Sema6A-plexinA4 signalling pathway in the developing vertebrate spinal cord, is necessary and sufficient to promote LMC motor neuron dendrite growth without affecting axons, and the Rho GEF activity of FARP1 is indispensable for this function [28]. In the silent state, the FERM and PH domains of FARP1 bind to the intracellular domain of plexinA4 independently of neuropilins. The binding of Sema6A to plexinA4 releases the autoinhibition of plexinA4 and activates the Rho GEF activity of FARP1. It is shown that plexinA4-dependent regulation of LMC motor neuron dendrite length requires ligand activation, and FARP1 is not dissociated from plexinA4 in the presence of Sema6A binding. Sema6A binding does not affect the binding affinity between plexinA4 and FARP1, but stimulates the Rho GEF activity of FARP1 towards downstream Rho GTPases. FARP1 Rho-GEF activity is critical for mediating the Sema6A-plexinA4-dependent growth of LMC motor neuron dendrites, but how it modifies the downstream effectors is still unclear [28]. As a Rho GEF, FARP1 might regulate downstream signalling pathways by modulating the activity of small Rho GTPase proteins. Rho GTPases signalling pathways are regulators of actin and microtubule cytoskeletons, which are the fundamental structures of dendrites. Thus, it is presumed that FARP1 modulates cytoskeletal structures by regulating Rho GTPases, and in turn promotes LMC motor dendrite growth.

Sema7A is a membrane-anchored semaphorin, acting as both an immune and a neural Semaphorin through its receptor plexinC1 and $\beta 1$-integrins [32-34]. In both the central and peripheral nervous system, Sema7A act as contractive guidance cue to enhance axon growth and it is also required for axon tract formation during embryonic development [32]. Sema7A activates plexinC1 by dimerization and in turn activates mitogen-activated protein kinase (MAPK), Lim Kinase II (a protein that phosphorylates cofilin), then inactivates cofilin (an actinbinding protein involved in cell migration) [32,33]. Sema7A also activates $\beta 1$-integrins, and in turn activates focal adhesion kinase (FAK), followed by a secondary MAPK activation [35]. It is known that LIMK is activated by p21-activated kinase 1(PAK1), which is in turn activated by Rac1. Thus it is highly possible that Rac1 is downstream of plexinC1 and/or $\beta 1$-integrins activation via Sema7A binding. It is great interesting to determine whether small Rho GTPases can interact with the intracellular domain of plexinC1 and have an influence on Sema7A-induced axon growth.

\section{RAS GTPASES AND SEMAPHORIN SIGNALLING OUTCOMES}

The activation of plexinA1 and plexinB1 by Sema3A and Sema4D respectively leads to the activation of R-Ras GAP activity of intracellular domain of plexinA1 and plexinB1 [10,22]. R-Ras activates the Phosphoinositide 3-kinase (PI3K) and generates Phosphatidy-linositol $(3,4,5)$-trisphosphate (PtdIns $(3,4,5)$ P3) second messenger, which alters the conformation and localization of AKT and leads its activation, promoting cell adhesion and migration through integrin activation [36] (Figure 1). In addition, the intracellular domain of plexins is highly conserved in all plexin family members. Thus it is possible that the intersection between plexin and Ras signalling is a common feature of plexin signalling. The Ras GTPases family is comprised of a large number of small GTPases, among them R-Ras, TC21 and M-Ras are homologues [37]. However, it has been shown that the downstream signalling pathway after MRas activation is different from the one downstream of R-Ras. M-Ras activation leads to ERK activation through binding to B-Raf, which is similar to the downstream signalling pathway of classical Ras [36] (Figure 1). Interestingly, Sema4D-plexinB1 singlling pathway has been identified as the first example to active the GAP activity of plexinB1 for both R-Ras and M-Ras [38]. However, the functions of R-Ras and M-Ras in neuronal development are quite different: R-Ras activity is increased in the stage when axons are elongated and specified, while M-Ras activity is enhanced during the period of dendritic development [39]. The suppression of M-Ras activity via plexinB1 Ras GAP domain is correlated with reduced dentritic growth and branching. Thus, pleixnB1 induces axonal growth cone collapse and dendrites repellence through its Ras-GAP activity for R-Ras and M-Ras, respectively [38]. It was reported that plexins fail to function as GAPs for R-Ras unless there is a sequential interaction of the cytoplasmic domain of plexins with two different Rho GTPases; Rac1 and Rnd1 $[10,22]$. The fully activation of plexins appears to be dependent on the binding of a Rho GTPase to the Rho binding domain (RBD) and Semaphorin to the extracellular region as well $[10,22]$. Intriguingly, there are further evidences for the role of Rho GTPases in Semaphorin-plexin signalling pathways which are contrary to the former research. It was demonstrated that purified cytoplasmic region of Plexins exhibit GAP activity for Rap1B and Rap2A but not for R-ras or M-Ras. It was shown that the Rho GTPases do not induce the dimerization of plexins and do not stimulate the RapGAP ac- 
tivity of plexinscyto in solution either (Rap is another member of the Ras GTPases family) [40,41]. The roles of Rho GTPases in Semaphorin-plexin signalling pathways may be involved in sequestering Rho GTPases from their downstream effectors, or destablizing the inactive dimmer of plexin formed on the cell surface, or helping reconstruct the spatial structure of the plexin cytoplasmic region which is helpful for plexin activation $[40,41]$.

\section{RHO GTPASES AND NEURITE DEVELOPMENT IN PC12 CELLS}

PC12 is a cell line derived from a pheochromocytoma of the rat adrenal medulla; they stop dividing and begin to differentiation and neurite outgrowth after stimulation with nerve growth factor (NGF). Thus they have been used as a model system for neuronal differentiation. Binding of NGF to its tyrosine kinase receptor Trk leads to neuronal differentiation through Ras-MAPK signalling pathway [42]. The three well-studied Rho GTPases RhoA, Rac1 and Cdc42 are all involved in neuronal differentiation in PC12 cells. Overexpression of Rho in PC12 cells induces neurite retraction and cell rounding through its downsteam effector ROCK, while neurite outgrowth promoted by Cdc42 and Rac is mediated through MRCK $\alpha$ [43]. The less-studied Rho GTPases, such as Rnd1, Rnd2, Rnd3 and RhoG, also have functions in regulating neuronal differentiation in PC12 cells. Overexpression of Rnd1 in PC12 cells induces neurite outgrowth in a Rac-dependent manner, and the downregulation of RhoA activity mediated by direct interaction between Rnd 1 and FRS2 $\beta$ is also involved in this process [44,45]. Rnd2 induces neurites formation in PC12 cells through its downstream effector Rapostlin in a GTP dependent manner [46-48]. Rnd3 stimulates neurite outgrowth in PC12 cells through inhibiting the activity of RhoA/ROCK1 signalling [49]. Overexpression of wild-type RhoG in PC12 cells induces neurite outgrowth in the absence of NGF, and it was shown that RhoG is acting as a key regulator in NGF-induced neurite outgrowth downstream of Ras and upstream of Rac and Cdc42 [50].

\section{DISCUSSIONS}

Given that multiple members of Rho GTPases have important roles in neuronal development, it will be of great interest to define the functions of the poorly-understood members of the family in neuronal differentiation. The Rif GTPase is a relatively recent addition to the Rho family; it is found only in chordates and displays a relatively low homology to other family members. Activated Rif was found to be an alternative trigger for the formation of actin stress fibers in epithelial cells through ef- fector mDia1 [51,52]. Unlike the classical stress fiber inducer RhoA, Rif does not raise ROCK activity in cells, instead Rif appears to regulate the localization of myosin light chain phosphorylation [51,52]. Activated Rif also triggers the formation of filopodia through its effector mDia2, and this signalling pathway is totally independent of the classical filopodia-formation pathway induced by Cdc42 and its effector WASP/ARP2/3 [53]. Intriguingly, recent work has shown that Cdc42 and Rif work together in the formation of dendritic spines, which develop from dendritic filopodia [54]. Rif is quite distantly related with the other Rho GTPases family members, but on the same branch as human RhoD that is shown to have important functions on inhibiting Semaphorin-plexin signalling by binding to the intracellular domain of plexins [24]. Investigation of the roles of the poorlydefined Rho GTPases, such as Rif, in axonal and dendritic development will shed light on the fine-tune regulation of neuronal differentiation system.

\section{CONCLUSION}

Semaphorin-plexin signalling is essential for neuronal development regulation, and the disorder of the pathways has been implicated in neurological diseases and cancer [55]. The RBDs of plexins binds to a group of Rho family GTPases, such as Rac1, Rnd1 and RhoD, facilitating the normal transduction of the signals from Semaphorinplexin to the downstream effectors $[10,12,22,24,56]$. It is high possible that other Rho GTPases also have the affinity to the RBD of plexins, and on the contrary, there should be other plexins existing to interact with Rho GTPases besides the plexins listed here. Modulating the activities of certain Rho family members and/or their GEFs, GAPs and DGIs may be developed as novel strategies in neurological diseases and cancer therapies.

\section{ACKNOWLEDGEMENTS}

This work was supported by the Program of Higher-level talents of Inner Mongolia University (SPH-IMU 30105-125128).

\section{REFERENCES}

[1] Govek, E.E., Newey, S.E. and Van Aelst, L. (2005) The role of the Rho GTPases in neuronal development. Genes and Development, 19, 1-49. doi:10.1101/gad.1256405

[2] Hall, A. and Lalli, G. (2010) Rho and Ras GTPases in axon growth, guidance, and branching. Cold Spring Harbor Perspectives in Biology, 2, a001818. doi:10.1101/cshperspect.a001818

[3] Luo, L. (2000) Rho GTPases in neuronal morphogenesis. Nature Reviews Neuroscience, 1, 173-180. doi:10.1038/35044547

[4] Luo, Y., Raible, D. and Raper, J.A. (1993) Collapsin: A protein in brain that induces the collapse and paralysis of 
neuronal growth cones. Cell, 75, 217-227. doi:10.1016/0092-8674(93)80064-L

[5] Tran, T.S., Kolodkin, A.L. and Bharadwaj, R. (2007) Semaphorin regulation of cellular morphology. Annual Review of Cell and Developmental Biology, 23, 263-292. doi:10.1146/annurev.cellbio.22.010605.093554

[6] Gherardi, E., et al. (2004) The sema domain. Current Opinion in Structural Biology, 14, 669-678. doi:10.1016/j.sbi.2004.10.010

[7] Koppel, A.M., et al. (1997) A 70 amino acid region within the semaphorin domain activates specific cellular response of semaphorin family members. Neuron, 19, 531537. doi:10.1016/S0896-6273(00)80369-4

[8] Neufeld, G. and Kessler, O. (2008) The semaphorins versatile regulators of tumour progression and tumour angiogenesis. Nature Reviews Cancer, 8, 632-645. doi:10.1038/nrc2404

[9] Ohta, K., et al. (1995) Plexin: A novel neuronal cell surface molecule that mediates cell adhesion via a homophilic binding mechanism in the presence of calcium ions. Neuron, 14, 1189-1199. doi:10.1016/0896-6273(95)90266-X

[10] Oinuma, I., et al. (2004) The Semaphorin 4D receptor plexin-B1 is a GTPase activating protein for R-Ras. Science, 305, 862-865. doi:10.1126/science.1097545

[11] Tong, Y., et al. (2007) Binding of Rac1, Rnd1, and RhoD to a novel Rho GTPase interaction motif destabilizes dimerization of the plexin-B1 effector domain. Journal of Biological Chemistry, 282, 37215-37224. doi:10.1074/jbc.M703800200

[12] Driessens, M.H., et al. (2001) Plexin-B semaphorin receptors interact directly with active Rac and regulate the actin cytoskeleton by activating Rho. Current Biology, 11, 339-344. doi:10.1016/S0960-9822(01)00092-6

[13] Vikis, H.G., Li, W. and Guan, K.L. (2002) The plexinB1/Rac interaction inhibits PAK activation and enhances Sema4D ligand binding. Genes and Development, 16, 836845. doi:10.1101/gad.966402

[14] Swiercz, J.M., et al. (2002) Plexin-B1 directly interacts with PDZ-RhoGEF/LARG to regulate RhoA and growth cone morphology. Neuron, 35, 51-63. doi:10.1016/S0896-6273(02)00750-X

[15] Artigiani, S., et al. (2003) Functional regulation of semaphorin receptors by proprotein convertases. Journal of Biological Chemistry, 278, 10094-11101. doi:10.1074/jbc.M210156200

[16] Takahashi, T. and Strittmatter, S.M. (2001) Plexina1 autoinhibition by the plexin sema domain. Neuron, 29, 429439. doi:10.1016/S0896-6273(01)00216-1

[17] Puschel, A.W. (2002) The function of neuropilin/plexin complexes. Advances in Experimental Medicine and Biology, 515, 71-80. doi:10.1007/978-1-4615-0119-0_6

[18] Chen, H., et al. (1997) Neuropilin-2, a novel member of the neuropilin family, is a high affinity receptor for the semaphorins Sema E and Sema IV but not Sema III. Neuron, 19, 547-559. doi:10.1016/S0896-6273(00)80371-2

[19] He, Z. and Tessier-Lavigne, M. (1997) Neuropilin is a receptor for the axonal chemorepellent Semaphorin III. Cell,

\section{0, 739-751. doi:10.1016/S0092-8674(00)80534-6}

[20] Jan, Y.N. and Jan, L.Y. (2003) The control of dendrite development. Neuron, 40, 229-242. doi:10.1016/S0896-6273(03)00631-7

[21] Kubo, T., et al. (2002) A novel FERM domain including guanine nucleotide exchange factor is involved in Rac signaling and regulates neurite remodeling. Journal of Neuroscience, 22, 8504-8513.

[22] Toyofuku, T., et al. (2005) FARP2 triggers signals for Sema3A-mediated axonal repulsion. Nature Neuroscience, 8, 1712-1719. doi:10.1038/nn1596

[23] Oinuma, I., Katoh, H. and Negishi, M. (2004) Molecular dissection of the semaphorin $4 \mathrm{D}$ receptor plexin-B1-stimulated R-Ras GTPase-activating protein activity and neurite remodeling in hippocampal neurons. Journal of Neuroscience, 24, 11473-11480. doi:10.1523/JNEUROSCI.3257-04.2004

[24] Zanata, S.M., et al. (2002) Antagonistic effects of Rnd1 and RhoD GTPases regulate receptor activity in Semaphorin 3A-induced cytoskeletal collapse. Journal of Neuroscience, 22, 471-477.

[25] Artigiani, S., et al. (2004) Plexin-B3 is a functional receptor for semaphorin 5A. EMBO Reports, 5, 710-714. doi:10.1038/sj.embor.7400189

[26] Li, X. and Lee, A.Y. (2010) Semaphorin 5A and plexinB3 inhibit human glioma cell motility through RhoGDIalpha-mediated inactivation of Rac1 GTPase. Journal of Biological Chemistry, 285, 32436-32445. doi:10.1074/jbc.M110.120451

[27] Xu, X.M., et al. (2000) The transmembrane protein semaphorin 6A repels embryonic sympathetic axons. Journal of Neuroscience, 20, 2638-2648.

[28] Zhuang, B., Su, Y.S. and Sockanathan, S. (2009) FARP1 promotes the dendritic growth of spinal motor neuron subtypes through transmembrane Semaphorin6A and PlexinA4 signaling. Neuron, 61, 359-372. doi:10.1016/j.neuron.2008.12.022

[29] Polleux, F., Morrow, T. and Ghosh, A. (2000) Semaphorin 3A is a chemoattractant for cortical apical dendrites. Nature, 404, 567-573. doi:10.1038/35007001

[30] Koyano, Y., et al. (1997) Molecular cloning and characterization of CDEP, a novel human protein containing the ezrin-like domain of the band 4.1 superfamily and the Dbl homology domain of Rho guanine nucleotide exchange factors. Biochemical and Biophysical Research Communications, 241, 369-375. doi:10.1006/bbrc.1997.7826

[31] Koyano, Y., et al. (2001) Chondrocyte-derived ezrin-like domain containing protein (CDEP), a rho guanine nucleotide exchange factor, is inducible in chondrocytes by parathyroid hormone and cyclic AMP and has transforming activity in NIH3T3 cells. Osteoarthritis Cartilage, 9, S64S68.

[32] Pasterkamp, R.J., et al. (2003) Semaphorin 7A promotes axon outgrowth through integrins and MAPKs. Nature, 424, 398-405. doi:10.1038/nature01790

[33] Liu, H., et al. (2010) Structural basis of semaphorinplexin recognition and viral mimicry from Sema7A and 
A39R complexes with PlexinC1. Cell, 142, 749-761. doi:10.1016/j.cell.2010.07.040

[34] Walzer, T., et al. (2005) Plexin C1 engagement on mouse dendritic cells by viral semaphorin A39R induces actin cytoskeleton rearrangement and inhibits integrin-mediated adhesion and chemokine-induced migration. Journal of Immunology, 174, 51-59.

[35] Scott, G.A., et al. (2009) Plexin C1, a receptor for semaphorin $7 \mathrm{a}$, inactivates cofilin and is a potential tumor suppressor for melanoma progression. Journal of Investigative Dermatology, 129, 954-963. doi:10.1038/jid.2008.329

[36] Kinbara, K., et al. (2003) Ras GTPases: Integrins' friends or foes? Nature Reviews. Molecular Cell Biology, 4, 767776. doi:10.1038/nrm1229

[37] Matsumoto, K., Asano, T. and Endo, T. (1997) Novel small GTPase M-Ras participates in reorganization of actin cytoskeleton. Oncogene, 15, 2409-2417. doi:10.1038/sj.onc.1201416

[38] Saito, Y., et al. (2009) Plexin-B1 is a GTPase activating protein for M-Ras, remodelling dendrite morphology. EMBO Reports, 10, 614-621. doi:10.1038/embor.2009.63

[39] Oinuma, I., Katoh, H. and Negishi, M. (2007) R-Ras controls axon specification upstream of glycogen synthase kinase-3beta through integrin-linked kinase. Journal of Biological Chemistry, 282, 303-318. doi:10.1074/jbc.M607979200

[40] Bos, J.L. and Pannekoek, W.J. (2012) Semaphorin signaling meets rap. Science Signaling, 5, pe6. doi:10.1126/scisignal.2002913

[41] Wang, Y., et al. (2012) Plexins are GTPase-activating proteins for rap and are activated by induced dimerization. Science Signaling, 5, ra6. doi:10.1126/scisignal.2002636

[42] Gross, I., et al. (2001) Mammalian sprouty proteins inhibit cell growth and differentiation by preventing ras activation. Journal of Biological Chemistry, 276, 4646046468. doi:10.1074/jbc.M108234200

[43] Chen, X.Q., et al. (1999) The myotonic dystrophy kinaserelated Cdc42-binding kinase is involved in the regulation of neurite outgrowth in PC12 cells. Journal of Biological Chemistry, 274, 19901-19905. doi:10.1074/jbc.274.28.19901

[44] Harada, A., Katoh, H. and Negishi, M. (2005) Direct interaction of Rnd1 with FRS2 beta regulates Rnd1-induced down-regulation of RhoA activity and is involved in fibroblast growth factor-induced neurite outgrowth in PC12 cells. Journal of Biological Chemistry, 280, 1841818424. doi:10.1074/jbc.M411356200
[45] Aoki, J., et al. (2000) Rnd1, a novel rho family GTPase, induces the formation of neuritic processes in PC12 cells. Biochemical and Biophysical Research Communications, 278, 604-608. doi:10.1006/bbrc.2000.3842

[46] Fujita, H., et al. (2002) Rapostlin is a novel effector of Rnd2 GTPase inducing neurite branching. Journal of Biological Chemistry, 277, 45428-45434. doi:10.1074/jbc.M208090200

[47] Kakimoto, T., Katoh, H. and Negishi, M. (2004) Identification of splicing variants of Rapostlin, a novel RND2 effector that interacts with neural Wiskott-Aldrich syndrome protein and induces neurite branching. Journal of Biological Chemistry, 279, 14104-14110. doi:10.1074/jbc.M312763200

[48] Negishi, M. and Katoh, H. (2005) Rho family GTPases and dendrite plasticity. Neuroscientist, 11, 187-191. doi:10.1177/1073858404268768

[49] Talens-Visconti, R., et al. (2010) RhoE stimulates neurite-like outgrowth in PC12 cells through inhibition of the RhoA/ROCK-I signalling. Journal of Neurochemistry, 112, 1074-1087. doi:10.1111/j.1471-4159.2009.06526.x

[50] Katoh, H., et al. (2000) Small GTPase RhoG is a key regulator for neurite outgrowth in PC12 cells. Molecular and Cellular Biology, 20, 7378-7387. doi:10.1128/MCB.20.19.7378-7387.2000

[51] Fan, L. and Mellor, H. (2012) The small Rho GTPase Rif and actin cytoskeletal remodelling. Biochemical Society Transactions, 40, 268-272. doi:10.1042/BST20110625

[52] Fan, L., et al. (2010) The small GTPase Rif is an alternative trigger for the formation of actin stress fibers in epithelial cells. Journal of Cell Science, 123, 1247-1252. doi:10.1242/jcs.061754

[53] Pellegrin, S. and Mellor, H. (2005) The Rho family GTPase Rif induces filopodia through mDia2. Current Biology, 15, 129-133. doi:10.1016/j.cub.2005.01.011

[54] Hotulainen, P., et al. (2009) Defining mechanisms of actin polymerization and depolymerization during dendritic spine morphogenesis. Journal of Cell Biology, 185, 323-339. doi:10.1083/jcb.200809046

[55] Capparuccia, L. and Tamagnone, L. (2009) Semaphorin signaling in cancer cells and in cells of the tumor microenvironment-Two sides of a coin. Journal of Cell Science, 122, 1723-1736. doi:10.1242/jcs.030197

[56] Rohm, B., et al. (2000) The semaphorin 3A receptor may directly regulate the activity of small GTPases. FEBS Letters, 486, 68-72. doi:10.1016/S0014-5793(00)02240-7 